\title{
Clinical Study \\ Skin and Soft Tissue Infections in Patients with Solid Tumours
}

\author{
Diamantis P. Kofteridis, ${ }^{1}$ Antonios Valachis, ${ }^{1}$ Eirini Koutsounaki, ${ }^{1}$ Sofia Maraki, ${ }^{2}$ \\ Eleni Mavrogeni, ${ }^{1}$ Foteini N. Economidou, ${ }^{1}$ Dimitra Dimopoulou, ${ }^{1}$ Kostas Kalbakis, ${ }^{3}$ \\ Vassilis Georgoulias, ${ }^{3}$ and George Samonis ${ }^{1}$
}

${ }^{1}$ Infectious Disease Unit, Department of Internal Medicine, University Hospital of Heraklion, 71110 Heraklion, Crete, Greece

${ }^{2}$ Department of Clinical Microbiology, University Hospital of Heraklion, 71110 Heraklion, Crete, Greece

${ }^{3}$ Department of Medical Oncology, University Hospital of Heraklion, 71110 Heraklion, Crete, Greece

Correspondence should be addressed to Diamantis P. Kofteridis, kofterid@med.uoc.gr

Received 23 October 2011; Accepted 8 December 2011

Academic Editor: Pranatharthi H. Chandrasekar

Copyright (C) 2012 Diamantis P. Kofteridis et al. This is an open access article distributed under the Creative Commons Attribution License, which permits unrestricted use, distribution, and reproduction in any medium, provided the original work is properly cited.

\begin{abstract}
Background. Skin and soft tissue infections (SSTIs) in cancer patients represent a diagnostic challenge, as etiologic diagnosis is often missing, and clinical assessment of severity is difficult. Few studies have described (SSTIs) in patients with solid tumours (STs). Patients and Methods. Records of patients with ST and SSTI, cared for at the University Hospital of Heraklion, from 2002 to 2006 were retrospectively studied. Results. A total of 81 episodes of SSTIs, occurring in 71 patients with ST, have been evaluated. Their median age was 65 years (34-82). The most common underlying malignancy was breast cancer in 17 patients (24\%). Most episodes (89\%) occurred in nonneutropenics. Cellulitis/erysipelas was the most common clinical presentation (56; 69\%). Bacterial cultures were possible in $29(36 \%)$ patients. All patients received antimicrobial therapy, while in 17 episodes (21\%) an incision and drainage was required. Treatment failure occurred in 20 episodes (25\%). Five patients (7\%) died due to sepsis. None was neutropenic. Severe sepsis on admission $(P=0.002)$ and prior blood transfusion $(P=0.043)$ were independent predictors of treatment failure. Conclusion. SSTIs can be life threatening among patients with ST. Early diagnosis and appropriate treatment are of the utmost importance, since sepsis was proven a significant factor of unfavourable outcome.
\end{abstract}

\section{Introduction}

Despite the advances in prevention and management of infectious complications in cancer patients, they still remain an important cause of morbidity and mortality [1-4]. Additionally, infections, especially in neutropenic patients, may lead to delay in delivering planned chemotherapy, and this event may have an impact upon patients' survival [4-7].

Cancer patients are prone and highly susceptible to infections through obstructive processes or immune system disorders due to the disease per se or cytotoxic therapy and/or disruption of the integrity of anatomic barriers due to chemotherapy, radiotherapy, or intravascular devices $[4,8,9]$.

Additionally, cancer patients are at risk of acquiring nosocomial infections, since they often undergo invasive diagnostic and therapeutic procedures, intravenous line placement, and hospitalization leading to the alteration of their skin and gut microbial flora $[4,10]$.
SSTIs in cancer patients represent a diagnostic challenge, as etiologic diagnosis is often missing, and clinical assessment of severity is difficult. Even small and innocuous in appearance, lesions should be carefully evaluated, because due to different degree of immunosuppression, the inflammatory response may be attenuated, and an apparently mild infection can become rapidly life threatening. Finally, SSTIs may be the result of a systemic disease spreading to the skin $[4,5,11]$.

Therefore, early recognition and prompt administration of appropriate empirical antimicrobial treatment are crucial, especially in the neutropenic host $[4,5]$.

Few studies, however, have described SSTIs in immunocompromised patients, including those with cancer and neutropenia [7-12].

The purpose of our study was to describe the clinical and microbiological characteristics of SSTIs in patients with STs and to determine factors leading to treatment failure. 


\section{Materials and Methods}

2.1. Setting. Records of patients with STs and SSTIs, cared for at the University Hospital of Heraklion, from 2002 to 2006 were retrospectively studied.

2.2. Ethical Approval. The study has been approved by the relevant committee of the University Hospital of Heraklion.

2.3. Study Design and Definitions. A SSTI was defined as community acquired if the episode developed in an outpatient setting or within $48 \mathrm{~h}$ after hospital admission in patients who did not fit the criteria for a healthcare-associated SSTI. Healthcare-associated SSTI was defined as the episode developing at the time of hospital admission or within $48 \mathrm{~h}$ after admission if the patient had been hospitalized or had been cared for at the day clinic of a hospital during the preceding 30 days [13]. Hospital-acquired SSTI was defined as the episode developing after the first 48 hours of hospital stay [13].

SSTIs were grouped as cellulitis or erysipelas, furuncles or carbuncles, cutaneous abscesses, central venous catheter exitsite infection, wound infections, pyomyositis, and herpes zoster. Diagnoses were defined using established criteria [5].

Sepsis, severe sepsis, and septic shock were also defined using established criteria [14]. In more details, sepsis was defined as the clinical syndrome that results from a dysregulated inflammatory response to an infection. It exists if two or more of the following abnormalities are present, along with either a culture-proven or visually identified infection: temperature $>38.5^{\circ} \mathrm{C}$ or $<35^{\circ} \mathrm{C}$, heart rate $>90$ beats $/ \mathrm{min}$, respiratory rate $>20$ breaths $/ \mathrm{min}$ or $\mathrm{PaCO} 2<32 \mathrm{mmHg}$, $\mathrm{WBC}>12,000$ cells $/ \mathrm{mm}^{3},<4000$ cells $/ \mathrm{mm}^{3}$, or $>10$ percent immature (band) forms.

Severe sepsis was defined as sepsis plus at least one of the following signs of hypoperfusion or organ dysfunction: areas of mottled skin, capillary refilling requiring three seconds or longer, urine output $<0.5 \mathrm{~mL} / \mathrm{kg}$ for at least one hour, or renal replacement therapy, lactate $>2 \mathrm{mmol} / \mathrm{L}$, abrupt change in mental status, abnormal electroencephalographic (EEG) findings, platelet count $<100,000$ platelets $/ \mathrm{mL}$, disseminated intravascular coagulation, acute lung injury or acute respiratory distress syndrome (ARDS), cardiac dysfunction (i.e., left ventricular systolic dysfunction), as defined by echocardiography or direct measurement of the cardiac index [14].

Patient's tumour response status to antitumour treatment at the date of onset of the SSTI episode was classified as complete response (CR), partial response (PR), stable disease $(\mathrm{SD})$, or progressive disease (PD).

The definition of neutropenia was in accordance with the NCI common toxicity criteria, version 2.0 [15].

Empirical antimicrobial treatment was considered appropriate if the organism isolated from the infected area was susceptible at least to one of the antimicrobials prescribed. In any other case, treatment was considered inappropriate, on the condition that the SSTI had been attributed to a known organism.

Treatment failure was defined by documented worsening of clinical signs and symptoms of the infection, such as recur- rence or worsening of fever, increased purulence, erythema, induration, and/or tenderness at least 2 days after treatment initiation, and if one of the following had happened: performance of a second, not previously planned, incision and drainage procedure, and/or readmission to the hospital due to the same SSTI episode and/or death due to septic shock.

2.4. Data Review. Patient's age, sex, underlying ST, blood transfusions, antimicrobial treatment, medications, exposure to radiotherapy or chemotherapy (during the preceding 30 days), and status of neoplastic disease (CR, PR, SD, $\mathrm{PD})$, performance status (PS) and any invasive procedure performed (during the preceding 10 days), date of onset of the episode, date of previous admission, results of complete blood count (CBC) (total white blood cell count and differential, hemoglobin level, and platelets), presence of fever, hypotension, tachycardia, tachypnea, shock, signs of localized infection as well as antimicrobial treatment administered for every episode were systematically recorded.

Microbiological information included the results of all relevant cultures, the source and type of isolated pathogen(s), and its (their) susceptibility pattern(s).

2.5. Statistical Analysis. Bivariate analyses were conducted by Pearson's chi-square test and Fisher's exact test, to compare categorical variables. For continuous variables, $t$-test and nonparametric Mann-Whitney test (for not normally distributed variables) were used. Variables with a $P$-value $<0.5$ were subsequently included in a stepwise logistic regression multivariate analysis, in order to determine independent risk factors for treatment failure. The SPSS for Windows software, version 16.0 (SPSS Inc., Chicago, IL, USA), was used for all statistical analyses.

\section{Results}

3.1. Patient Characteristics. Eighty-one episodes of SSTIs occurring in 71 patients were identified. The median age of patients was 65 (range 34-82) years. There were 38 males $(53.5 \%)$. The most common underlying malignancy was breast cancer in 17 patients (24\%), followed by colon in 14 (20\%) lung in $13(18 \%)$, genital in $10(14 \%)$, head and neck in $5(7 \%)$, urinary tract in $4(6 \%)$, and sarcoma in $3(4 \%)$ (Table 1).

At the onset of the SSTI 3 patients had a CR (5\%), 2 had a PR (2.5\%), 29 had SD (38\%), and 47 had PD (58\%). At the time of the episode, 24 patients (34\%) had a PS: 0, 41 ( $58 \%)$ PS: 1,12 (17\%) PS: 2, and 4 (5.5\%) PS 3.

Fifty out of 81 SSTI episodes $(62 \%)$ were healthcare-associated, $25(35 \%)$ community, and $6(7 \%)$ hospital acquired.

A central venous catheter was present in 15 episodes (18.5\%), blood had been transfused during the preceding 10 days in $21(26 \%)$, and invasive procedure was recorded in $30(37 \%)$. Chemotherapy and radiotherapy during the preceding 30 days had been administered in 52 (64\%) and 19 $(23.5 \%)$ episodes, respectively, and antimicrobial treatment in $30(37 \%)$.

Neutropenia was present only in 9 episodes (11\%), being grade IV in 4 , with median duration of 4 days (3-21). 
TABLe 1: Patients' characteristics.

\begin{tabular}{lc}
\hline Characteristics & No of patients (\%) \\
\hline Sex of patients & $38(53.5 \%)$ \\
Male & $33(46.5 \%)$ \\
Female & $65[34-82]$ \\
Median age of patients [range] & \\
Underlying malignancy & $17(24 \%)$ \\
Breast & $14(20 \%)$ \\
Colorectal & $13(18 \%)$ \\
Lung & $10(14 \%)$ \\
Genital & $5(7 \%)$ \\
Head \& neck & $4(6 \%)$ \\
Urinary tract & $3(4 \%)$ \\
Sarcoma & $2(3 \%)$ \\
Hepatobiliary & $1(1.3 \%)$ \\
Pancreas & $1(1.3 \%)$ \\
Stomach & $1(1.3 \%)$ \\
Unknown primary &
\end{tabular}

TABLE 2: Risk factors of the SSTIs episodes.

\begin{tabular}{lc}
\hline Factor & Episodes $(\%)$ \\
\hline Recent chemotherapy & $52(64)$ \\
Prior invasive procedure & $30(37)$ \\
Previous antimicrobial therapy & $30(37)$ \\
Prior blood transfusion & $21(26)$ \\
Recent radiotherapy & $19(23.5)$ \\
Central venous catheter & $15(18.5)$ \\
Neutropenia & $9(11)$ \\
\hline
\end{tabular}

Factors potentially associated with the infectious episodes are summarized in Table 2.

3.2. Clinical Presentation. Cellulites/erysipelas were the most common presentations (44 episodes; 54\%), followed by abscesses $(18 ; 22 \%)$ and exit-site infections $(8 ; 10 \%)$. Regarding the site of infection, SSTIs were more frequently located on the trunk $(28 ; 35 \%)$ followed by the genital and/or perirectal region $(19 ; 23 \%)$.

Considering signs and symptoms, erythema was present in 59 episodes (70\%), swelling in 41 (51\%) and localized pain in $34(42 \%)$. At the time of admission, fever was present in 52 (64\%) with median duration 3.5 days (1-14).

All the surgical site infections were wound infections while in all catheter-related infections, catheters had been removed.

Sepsis was present on admission in 43 episodes (53\%) and severe sepsis in $15(18.5 \%)$, while among the latter episodes $5(4 \%)$ were complicated by septic shock. Clinical characteristics of the episodes are summarized in Table 3.

3.3. Microbiology. Pathogens were isolated from skin and/or soft tissue lesions in 29 episodes (36\%). One pathogen was isolated from each of the 23 episodes, while two from
TABLE 3: Clinical characteristics of SSTI episodes.

\begin{tabular}{lc}
\hline Characteristic & No of episodes $(\%)$ \\
\hline Type of SSTI & \\
Cellulitis/erysipelas & $44(54)$ \\
Abscess & $18(22)$ \\
Exit-site infection & $8(10)$ \\
Wound infection & $5(6)$ \\
Herpes zoster & $4(5)$ \\
Furuncles/carbuncles & $1(1)$ \\
Pyomyositis & $1(1)$ \\
Site of infection & \\
Head and neck & $10(12)$ \\
Trunk & $28(35)$ \\
Upper extremities & $11(14)$ \\
Lower extremities & $13(16)$ \\
Genitoperineal/perirectal & $19(23)$ \\
Signs and symptoms & \\
Erythema & $59(70)$ \\
Fever & $52(64)$ \\
Swelling & $41(51)$ \\
Localized pain & $34(42)$ \\
Sepsis & $43(53)$ \\
Severe sepsis & $15(18.5)$ \\
Septic shock & $3(4)$ \\
\hline
\end{tabular}

TABLE 4: Microorganisms isolated from 29 SSTIs episodes.

\begin{tabular}{lc}
\hline Pathogens & Total no of isolates \\
\hline Gram negative & 7 \\
Pseudomonas aeruginosa & 7 \\
Escherichia coli & 2 \\
Klebsiella pneumoniae & 2 \\
Enterobacter cloacae & 1 \\
Citrobacter freundii & \\
Gram positive & 6 \\
Enterococcus faecalis & 5 \\
Staphylococcus epidermidis & 3 \\
Staphylococcus aureus & 1 \\
Enterococcus faecium & \\
Anaerobes & 1 \\
Bacteroides fragilis & 35 \\
\hline Total & \\
\hline
\end{tabular}

the remaining 6. Gram-negative bacteria were isolated in 13 (45\%), with Escherichia coli (6 out of 13;46\%) and Pseudomonas aeruginosa $(4 ; 31 \%)$ being the most frequent, followed by Enterobacter cloacae $(2 ; 15 \%)$ and Klebsiella pneumoniae $(1 ; 8 \%)$. Gram-positive organisms were isolated in 9 episodes (11\%) with Staphylococcus epidermidis being the most common (4 out of 9; 45\%) followed by Enterococcus faecalis $(3 ; 33 \%)$ and Staphylococcus aureus $(2 ; 22 \%)$. The isolated pathogens isolated are shown in Table 4.

Blood cultures were performed in all patients upon admission. No pathogens were isolated from blood. 
TABle 5: Characteristics of 5 patients with fatal outcome.

\begin{tabular}{ccccccc}
\hline Age & Sex & Underlying malignancy & Type of infection & Isolated organisms & Absolute neutrophils count & Initial inappropriate therapy \\
\hline 76 & F & Colorectal & Cellulitis & Escherichia coli & 15000 & Yes \\
64 & M & Lung & Abscess & polymicrobial & 18100 & Yes \\
82 & F & Breast & Abscess & negative & 4700 & - \\
48 & M & Lung & Cellulitis & Escherichia coli & 10100 & Yes \\
56 & M & Colorectal & Abscess & Escherichia coli & 29500 & Yes \\
\hline
\end{tabular}

3.4. Therapy. All patients received antimicrobial therapy, while in 17 episodes $(21 \%)$ an incision and drainage were required.

Initial antimicrobial treatment was considered inappropriate in 3 out of 20 episodes (15\%) treated successfully and in 7 out of $9(78 \%)$ treated unsuccessfully $(P=0.001)$.

3.5. Outcome and Predictors of Failure. Treatment failure occurred in 20 episodes (25\%). An additional drainage procedure was necessary in 8 . Seven episodes recurred with subsequent hospital admission and improved after treatment with second-line antimicrobial regimens. Five patients had a fatal outcome due to septic shock. Four of them have been inappropriately treated, and none was neutropenic. The overall mortality reached $7 \%$. Characteristics of the 5 patients who died are shown in Table 5.

In univariate analysis, three factors were found to have statistically significant association with treatment failure: prior blood transfusion, presence of severe sepsis on admission, and hospital-acquired or health-care associated infection.

Multivariate logistic regression analysis indicated that presence of severe sepsis on admission (OR 21.921, 95\% CI: 2.970-161.815; $P=0.002$ ) and prior blood transfusion (OR 4.460, 95\% CI: $1.049-18.954 ; P=0.043$ ) were independent predictors of treatment failure.

\section{Discussion}

The present study showed that SSTIs can be life threatening in patients with ST even in the absence of neutropenia. The majority of SSTIs were health-care associated, with gramnegative bacteria being most commonly isolated.

Cancer patients are at increased risk of infections, including SSTIs, due to multiple factors such as immunosuppression resulting from chemotherapy or due to the malignancy itself, impairment of normal leukocyte function, disruption of anatomical barriers, or obstructive phenomena $[2-5,7,16]$.

SSTIs in patients with STs may have different etiology than those occurring in the general population and variable clinical presentations due to different degrees of immunosuppression and type of neoplasia. Additionally, a broad range of differential diagnoses that may mimic skin infections should be taken in consideration such as drug and transfusion-associated rashes, hemorrhagic areas, and malignancies.
In patients with tumours, a normally mild SSTI can be rapidly transformed into a life-threatening disease or can be a manifestation of a serious systemic disease spreading to the skin $[4,5]$. Furthermore, SSTIs, especially in neutropenic patients, may delay the delivery of planned chemotherapy with impact on the outcome of the underlying malignancy [6].

Although infectious complications in patients with STs have been well described, specific data on SSTIs are rare and inconclusive $[4,5,7-12]$.

The present results indicate that SSTIs can be life-threatening among patients with STs, even in the absence of neutropenia. Neutropenia remains an important risk factor for infection in patients with cancer although in the present study only a limited number of patients were neutropenic $[2,16,17]$.

Additionally cancer patients are at increased risk of nosocomial infections since they often undergo invasive procedures, intravenous line placement, and hospitalizations leading to the alteration of their microbial flora [17]. Indeed the present study revealed that $62 \%$ of the episodes were health care associated while an additional $7 \%$ was pure nosocomial. This is not surprising considering the fact that most cancer patients nowadays, including the present ones, receive routine care in day clinics as outpatients $[16,17]$.

In the present study, cellulitis and erysipelas were the most common clinical presentations. Microorganisms were isolated from lesions in 36\% of the episodes with gramnegative pathogens being the most common bacteria isolated and this is in contrast with other studies in the general population in which gram-positive organisms, Staphylococcus aureus and Streptococcus pyogenes, are the predominant pathogens $[18,19]$. There are, however, studies in accordance with our results indicating that gram-negative bacteria play an important role as causative agents of skin and soft-tissue infections in many areas of the world, especially in patients with liver cirrhosis, malignancy, and alcoholism $[20,21]$.

It is worth noting that none of the 71 patients had positive blood cultures. Two recent literature reviews concluded that blood cultures are not necessary in acute cellulitis in immunocompetent hosts $[22,23]$. Although the present results are negative, the question whether blood cultures are helpful in immunocompromised patients with SSTIs has not yet been answered [5].

The role of antimicrobial treatment of SSTIs is also not fully clarified. There are reports describing cures of immunocompetent patients receiving inappropriate or no treatment at all [24]. Furthermore, literature does not elucidate if inappropriate antimicrobial treatment in cancer patients 
with SSTIs plays a crucially negative role, considering also the fact that may delay the antitumour therapy due to prolonged infection [6]. However, in the present study, inappropriate initial antibiotic therapy was significantly associated with treatment failure. Additionally, four out of five patients with fatal outcome had received inappropriate treatment.

Initial empirical antibiotic selection should be based on severity stratification of SSTIs and clinical care guidelines [5]. In stable pts with skin/soft tissue infections empiric therapy is usually aimed against gram-positive pathogens. However, as health-care-associated infections occur often among patients with solid tumors, empirical treatment with antimicrobial agents covering gram-negative bacteria should also be considered.

Factors such as recent chemotherapy, prior invasive procedures, previous antimicrobial treatment, and prior blood transfusion are commonly associated with SSTIs in patients with STs $[4,5]$.

Multivariate analysis revealed that only two clinical factors had a statistically significant association with treatment failure: the occurrence of severe sepsis and the prior blood transfusion.

Severe sepsis is an already known important complication in cancer patients developing infections that carries a high mortality rate [25]. The rate of sepsis occurring in the present patients was high. No other source except the SSTIs could be implicated for the septic episodes. This finding indicates the high risk of this type of infections in the present patients' population.

The clinical significance of immune suppression secondary to blood transfusion in cancer patients remains controversial. Adverse effects of blood transfusions have been documented in patients undergoing colorectal and gastrointestinal surgery as demonstrated by higher infection rates among them $[26,27]$. On the contrary, a meta-analysis that examined perioperative allogenic blood transfusion in surgical oncology patients concluded that there was no evidence to support adverse infectious sequelae [28].

SSTIs are usually curable [5]. However, in the present study, mortality reached $7 \%$. A great number of the present population presented with severe and life-threatening infections. This can be explained by the fact that many infectious episodes were healthcare associated or nosocomial.

Although 5 patients died due to septic shock that was related to some degree to their infection, no organisms were isolated from blood. It is of note that the disease's status and bad performance status did not affect significantly outcome. This is probably due to the fact that a relatively small number of patients had a PS $\geq 2$.

In conclusion, the majority of SSTIs in cancer patients of the present study were healthcare associated, with gramnegative bacteria being most commonly isolated from the infected sites. Furthermore, the study showed that SSTIs can be life threatening in this patients' population, even in the absence of neutropenia. Early diagnosis is of utmost importance, since sepsis on admission has been proven a significant factor of unfavourable outcome. Although $7 \%$ of the patients died due to septic shock that was related to some degree to their infections, no organisms were isolated from blood.

\section{Acknowledgment}

This research was presented in part at 19th European Congress of Clinical Microbiology and Infectious Diseases, Helsinki, Finland, 16-19 May 1, 2009.

\section{References}

[1] T. J. Smith, J. Khatcheressian, G. H. Lyman et al., “2006 Update of recommendations for the use of white blood cell growth factors: an evidence-based clinical practice guideline," Journal of Clinical Oncology, vol. 24, no. 19, pp. 3187-3205, 2006.

[2] A. G. Freifeld, E. J. Bow, K. A. Sepkowitz et al., "Executive summary: clinical practice guideline for the use of antimicrobial agents in neutropenic patients with cancer: 2010 update by the infectious diseases society of America," Clinical Infectious Diseases, vol. 52, no. 4, pp. 427-431, 2011.

[3] E. Robenshtok, A. Gafter-Gvili, E. Goldberg et al., "Antifungal prophylaxis in cancer patients after chemotherapy or hematopoietic stem-cell transplantation: systematic review and meta-analysis," Journal of Clinical Oncology, vol. 25, no. 34, pp. 5471-5489, 2007.

[4] P. A. Pizzo, J. Meyers, A. G. Freifeld, and T. Walsh, "Infection in the cancer patient," in Cancer Principle and Practice of Oncology, V. T. DeVita, S. Hellman, and S. Rosenberg, Eds., pp. 2292-2337, Lippincot Company, Philadelphia, Pa, USA, 8th edition, 2009.

[5] D. L. Stevens, A. L. Bisno, H. F. Chambers et al., "Practice guidelines for the diagnosis and management of skin and softtissue infections," Clinical Infectious Diseases, vol. 41, no. 10, pp. 1373-1406, 2005.

[6] S. Khan, A. Dhadda, D. Fyfe, and S. Sundar, "Impact of neutropenia on delivering planned chemotherapy for solid tumours: original article," European Journal of Cancer Care, vol. 17, no. 1, pp. 19-25, 2008.

[7] E. Toussaint, E. Bahel-Ball, M. Vekemans et al., "Causes of fever in cancer patients (prospective study over 477 episodes)," Supportive Care in Cancer, vol. 14, no. 7, pp. 763-769, 2006.

[8] D. Yadegarynia, J. Tarrand, I. Raad, and K. Rolston, "Current spectrum of bacterial infections in patients with cancer," Clinical Infectious Diseases, vol. 37, no. 8, pp. 1144-1145, 2003.

[9] M. Kamana, C. Escalante, C. A. Mullen, S. Frisbee-Hume, and K. V. I. Rolston, "Bacterial infections in low-risk, febrile neutropenic patients: over a decade of experience at a comprehensive cancer center," Cancer, vol. 104, no. 2, pp. 422426, 2005.

[10] I. R. O. Novakova, J. P. Donnelly, and B. De Pauw, "Potential sites of infection that develop in febrile neutropenic patients," Leukemia and Lymphoma, vol. 10, no. 6, pp. 461-467, 1993.

[11] F. A. Lopez and C. V. Sanders, "Dermatologic infections in the immunocompromised(non-HIV) host," Infectious Disease Clinics of North America, vol. 15, no. 2, pp. 671-702, 2001.

[12] J. S. Wolfson, A. J. Sober, and R. H. Rubin, "Dermatologic manifestations of infections in immunocompromised patients," Medicine, vol. 64, no. 2, pp. 115-133, 1985.

[13] T. C. Horan, M. Andrus, and M. A. Dudeck, "CDC/NHSN surveillance definition of health care-associated infection and criteria for specific types of infections in the acute care setting," American Journal of Infection Control, vol. 36, no. 5, pp. 309332, 2008.

[14] Members of the American College of Chest Physicians, "American College of Chest Physicians/ Society of Critical Care Medicine consensus conference: definitions for sepsis and organ 
failure and guidelines for the use of innovative therapies in sepsis," Critical Care Medicine, vol. 20, pp. 864-874, 1992.

[15] National Cancer Institute, "Common Toxicity Criteria (NCICTC) v2.0," http://ctep.cancer.gov/protocolDevelopment/electronic_applications/docs/ctcv20_4-30-992.pdf.

[16] S. Vento and F. Cainelli, "Infections in patients with cancer undergoing chemotherapy: aetiology, prevention, and treatment," Lancet Oncology, vol. 4, no. 10, pp. 595-604, 2003.

[17] S. H. Sutton and J. P. Flaherty, "Infections associated with solid tumors," Cancer treatment and research, vol. 96, pp. 105-141, 1998.

[18] E. W. Hook III, T. M. Hooton, and C. A. Horton, "Microbiologic evaluation of cutaneous cellulitis in adults," Archives of Internal Medicine, vol. 146, no. 2, pp. 295-297, 1986.

[19] T. Duvanel, R. Auckenthaler, P. Rohner, M. Harms, and J. H. Saurat, "Quantitative cultures of biopsy specimens from cutaneous cellulitis," Archives of Internal Medicine, vol. 149, no. 2, pp. 293-296, 1989.

[20] G. J. Moet, R. N. Jones, D. J. Biedenbach, M. G. Stilwell, and T. R. Fritsche, "Contemporary causes of skin and soft tissue infections in North America, Latin America, and Europe: report from the SENTRY Antimicrobial Surveillance Program (1998-2004)," Diagnostic Microbiology and Infectious Disease, vol. 57, no. 1, pp. 7-13, 2007.

[21] C. M. Chang, H. C. Lee, N. Y. Lee et al., "Communityacquired Klebsiella pneumoniae complicated skin and softtissue infections of extremities: emphasis on cirrhotic patients and gas formation," Infection, vol. 36, no. 4, pp. 328-334, 2008.

[22] A. M. Mills and E. H. Chen, "Are blood cultures necessary in adults with cellulitis?" Annals of Emergency Medicine, vol. 45, no. 5, pp. 548-549, 2005.

[23] A. Stevenson, P. Hider, and M. Than, "The utility of blood cultures in the management of non-facial cellulitis appears to be low," New Zealand Medical Journal, vol. 118, no. 1211, pp. 1351-1357, 2005.

[24] K. Z. Paydar, S. L. Hansen, E. D. Charlebois, H. W. Harris, and D. M. Young, "Inappropriate antibiotic use in soft tissue infections," Archives of Surgery, vol. 141, no. 9, pp. 850-854, 2006.

[25] M. D. Williams, L. A. Braun, L. M. Cooper et al., "Hospitalized cancer patients with severe sepsis: analysis of incidence, mortality, and associated costs of care," Critical Care, vol. 8, no. 5, pp. R291-R298, 2004.

[26] M. Braga, A. Vignali, G. Radaelli, L. Gianotti, and V. Di Carlo, "Association between perioperative blood transfusion and postoperative infection in patients having elective operations for gastrointestinal cancer," European Journal of Surgery, vol. 158, no. 10, pp. 531-536, 1992.

[27] C. D. Morris, K. Sepkowitz, C. Fonshell et al., "Prospective identification of risk factors for wound infection after lower extremity oncologic surgery," Annals of Surgical Oncology, vol. 10, no. 7, pp. 778-782, 2003.

[28] F. A. McAlister, H. D. Clark, P. S. Wells, and A. Laupacis, "Perioperative allogeneic blood transfusion does not cause adverse sequelae in patients with cancer: a meta-analysis of unconfounded studies," British Journal of Surgery, vol. 85, no. 2, pp. 171-178, 1998. 


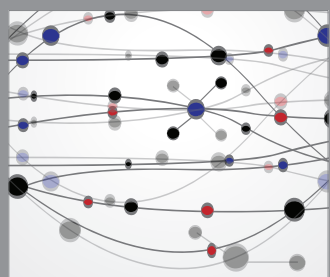

The Scientific World Journal
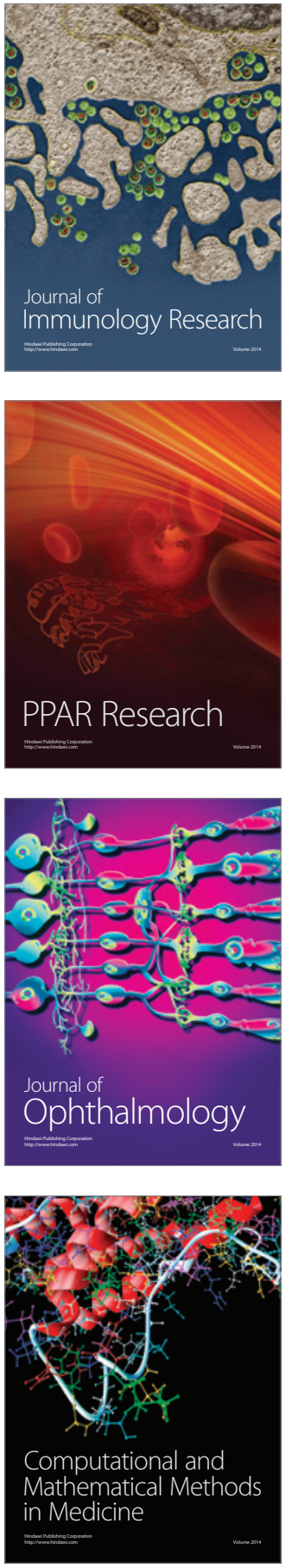

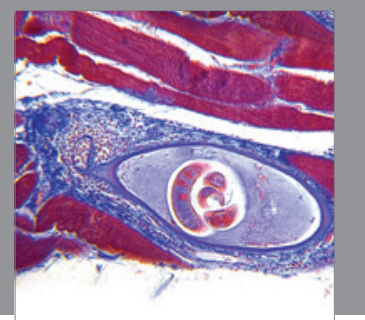

Gastroenterology

Research and Practice
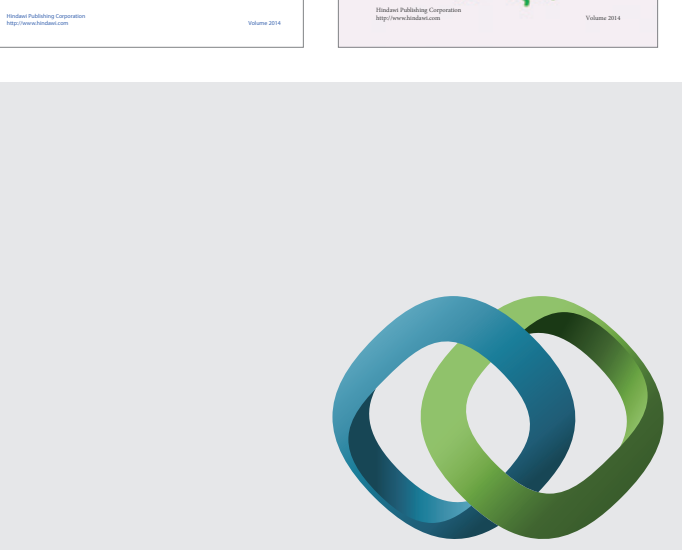

\section{Hindawi}

Submit your manuscripts at

http://www.hindawi.com
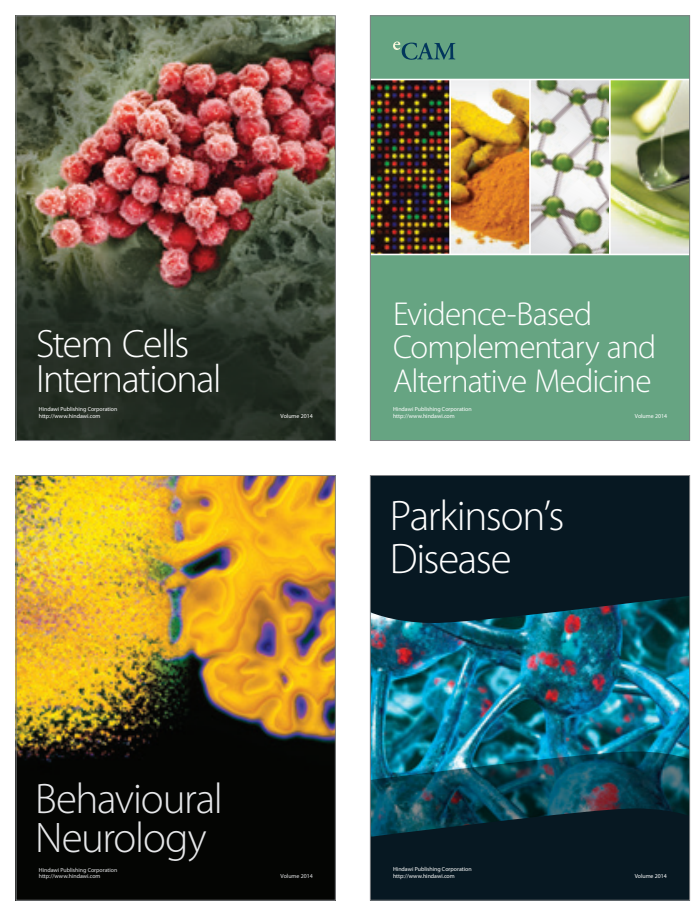

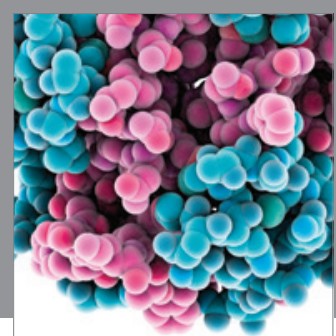

Journal of
Diabetes Research

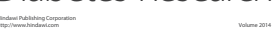

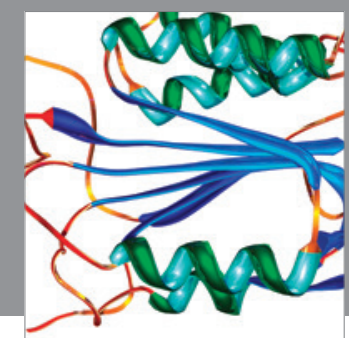

Disease Markers
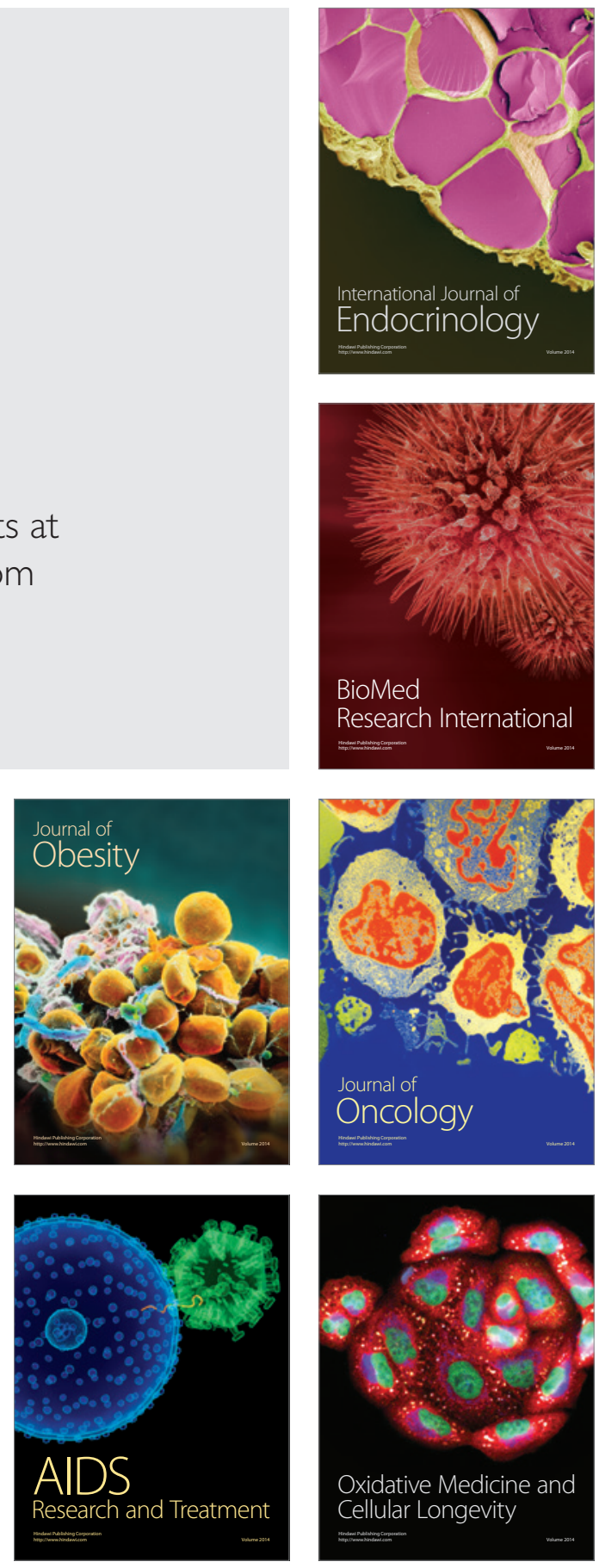